\title{
И.В. Демичев
}

\section{ДИНАМИКА СЛОЖНОЙ СИСТЕМЫ ИДЕНТИЧНОСТЕЙ ${ }^{1}$}

\begin{abstract}
В данной статье на основании теоретической модели сложной системы идентичностей рассматривается проблема динамики отношений между ними. Выделяются три уровня анализа динамики, образуемые фундаментальными, частными и актуальнымм группами факторов, влияющих на социокультурную динамику. На каждом уровне формулируются по три возможных общих направления динамики, характеризующих состояние отношений между идентичностями системы.

Ключевые слова: идентичность, динамика идентичности, конструктивизм.
\end{abstract}

В современной гуманитарной науке тема коллективной идентичности и ее динамики представляет интерес для многих. Большинство исследователей сходятся во мнении о неустойчивом состоянии российской идентичности, связанном с противоречиями как в содержании (различия в оценках советского и дореволюционного прошлого; различия в региональном и профессионально-стратовом аспектах), так и в соотношении различных компонентов (политико-идеологического, этнического, конфессионального и т.д.) $[1,2]$. В свете данных дискуссий считаем целесообразным проанализировать основные факторы динамики идентичности. В работе идентичности рассматриваются в рамках теории конструктивизма как некие социальные конструкты [3], формирующие сложную систему коллективных идентичностей, в которой выделяются типы идентичностей (территориальные, профессиональностратовые, национальные, конфессиональные) и виды (фокусные, вторичные, атрибутивные). Отношения между этими идентичностями и распределение по видам системы являются динамичными, и возможен их переход из одного вида в другой. На основе данных отношений формируются режимы функционирования идентичностей, которые сводятся к стабильному и нестабильному режимам. При нестабильном режиме функционирования может быть режим акцентированной идентичности и режим инерционной идентичности. В первом случае идентичности присваивается обязательный статус, а при втором режиме идентичность сводится в большинстве своем лишь к внешней атрибутике. Данные установки могут быть и внутри одной идентичности, и в соответствии с тем, какие установки преобладают в нем, формируется общий режим функционирования этой идентичности. Отношения между элементами системы зависят от этих установок, комплементарные и взаимные установки приводят к гармоничному состоянию системы идентичностей, некомплементарность и невзаимность приводят к установлению напряженности и конфликтности отношений между данными видами коллективных идентичностей и всей системы в целом.

\footnotetext{
${ }^{1}$ Работа подготовлена в рамках исполнения Государственного задания «Этноконфессиональный фактор в формировании российской гражданской идентичности в Республике Башкортостан».
} 
На основании этого можно перейти к описанию динамики системы идентичностей. Существует огромный соблазн свести ее к количественной оценке самоопределений по национальному и конфессиональному признаку, а также к описанию работы соответствующих организаций, однако этого совершенно недостаточно. Динамика идентичностей, по понятным основаниям, касается не только самоназвания: все их элементы (образы, маркеры, стереотипы поведения, формы солидарности) подвержены изменениям, которые зависят как от состояния социокультурной общности, так и от коллективного и группового самосознания ее членов, а кроме того, от функционирования специализированных и неспециализированных институционально-дискурсивных структур, влияющих на идентичность, коллективное и групповое сознание и общность в целом. Однако в этом случае проблема динамики идентичностей оказывается слишком размытой и не поддается рациональному анализу.

Можно выделить три группы факторов динамики, сказывающихся на системе идентичностей данной социокультурной общности, каждая из которых отвечает за логику и направленность изменений на своем уровне.

В первую очередь это группа факторов, которая отвечает за основные процессы, протекающие в общности в целом, обозначим их как фундаментальные. Здесь отмечаются наиболее общие тенденции развития отношений институциональных и дискурсивных, определяющие не конкретику, а общие условия, в которых существуют и функционируют идентичности, а также обеспечивающие их структуры, от них зависят как общее состояние аудиторий и сообществ, репрезентирующих себя и отвечающих на репрезентацию, так и более конкретные формы, например эстетические и когнитивные, которые обусловливают общее состояние индивидуального, группового и коллективного сознания всех членов общности.

Во вторую очередь это группа факторов, которая касается существования, деятельности и трансформаций входящих в данную общность сообществ в данном случае их самих, поскольку во многом формы и функционирование идентичностей определяются не столько отвлеченными установками, сколько реальностью и рефлексией отношений между сообществами и внутри них. Соответственно, факты отношений между ними, между подгруппами сообществ и т.п. способны направить в ту или иную сторону процесс воспроизводства идентичности, а также влияют на внутреннее их членение. Эту группу факторов можно обозначить как частную.

Наконец, в третью очередь это группа факторов, которая касается уже непосредственно институционально-дискурсивных структур сообществ и общности в целом, в рамках которых происходит формирование, рефлексия, трансляция и актуализация установок, образов, маркеров и других элементов идентичностей, а также реагирование на это со стороны данной и всех остальных аудиторий носителей идентичностей, членов сообществ. Это наиболее активный и изменчивый уровень рассмотрения динамики системы идентичностей, поскольку здесь соответствующие вопросы наиболее наглядно выражены, хотя и вряд ли представляют собой некоторую целостность. В то же время именно факторы данной группы наиболее подвержены оперированию. Соответственно, этот уровень имеет смысл обозначить как актуальный. 
Динамика


Рис. 1. Факторы и тенденции динамики идентичностей

Необходимо отметить, что эти три группы факторов нельзя рассматривать отдельно друг от друга: это уровни анализа динамики идентичностей, каждый из которых как определяется другими, так и оказывает на них влияние. Так, фундаментальный уровень, с одной стороны, определяет саму логику и генеральную тенденцию, в рамках которой могут развиваться эффекты частного уровня, выражаемые в деятельности организаций и структур, - и все вместе они определяют динамику системы идентичностей, образуя некоторую равнодействующую. С другой стороны, в зависимости от того, как отрефлексированы и выражены установки идентичностей и отношения между ними в среде актуальных структур, а также на какие их варианты склонны ориентироваться широкие аудитории сообществ данной социокультурной общности, зависит, какие отношения между ними будут проявляться на уровне частных факторов, а уже установки, зафиксированные здесь, оказывают влияние на протекающие в общности фундаментальные процессы. В этом смысле вектор зависимости «от фундаментального к актуальному» отражает объективный (интерсубъективный) характер, а вектор зависимости «от актуального к фундаментальному» - оперативный.

Не вдаваясь в подробный анализ самих групп выделенных факторов, которые носят дискуссионный и проблематичный характер, можно отметить варианты образуемых ими тенденций, которые будут сказываться на специфике функционирования системы идентичностей социокультурной общности и ее сообществ.

На фундаментальном уровне можно выделить, во-первых, стабильное состояние системы, когда ее институционально-дискурсивные структуры устойчивы, их изменения не выходят за пределы самопогашаемых флуктуаций, а сообщества, организованные ими, успешно воспроизводятся и не входят в конфликт друг с другом. В этом случае и система идентичностей будет проявлять устойчивость, гася нежелательные изменения за счет собственных механизмов поддержания порядка, а сама установка на изменения не будет рассматриваться носителями как правомочная. Во-вторых, это нестабильные состояния общности, которые можно подразделить на два существенно отличающихся друг от друга вида. Следует отметить, что вне зависимости от них нестабильный режим функционирования общности в любом случае будет реализовывать установки на изменения, возможности системы по подавле- 
нию изменений будут исчерпаны, а флуктуации, входя во взаимный резонанс, будут приобретать устойчивый характер. Оба эти режима отличаются друг от друга общей направленностью изменений, но по итогам предполагают некоторую новую форму снятых противоречий между элементами системы, ложащуюся в основу некоторого нового стабильного режима функционирования общности.

Различия между ними касаются направленности изменений на расширение возможностей системы или, наоборот, их сокращение - создание таких структур и отношений, которые окажутся в состоянии генерировать, распределять и употреблять больший или меньший объем материальных, социальных и культурных благ. Поэтому эти процессы можно обозначить как модернизационный в первом случае или архаизирующий - во втором. Следует оговориться, что в любом случае при нестабильном режиме функционирования общности будут проявляться интенции обоих направлений [4], поскольку они вызываются попытками адаптации системы к изменившимся условиям существования общности, а значит, связаны с перераспределением благ в ее рамках. При этом части структур и областей общности будут по-разному обеспечиваться ими - и там, где благ будет недостаточно для воспроизводства структуры отношений, будут развиваться архаизирующие процессы, а там, где благ будет достаточно, будут развиваться процессы модернизационные. В таких частях социокультурной общности будут также по-разному разворачиваться и процессы трансформации идентичностей, что в значительной мере усложняет анализ общей ситуации и неизбежно порождает дополнительные напряженности в их системе. Общую же оценку состояния социокультурной общности можно давать, ориентируясь на сравнительную мощность обеих тенденций - смотря по тому, какая из них может и способна лечь в основу предполагаемой будущей стабильности.

Для примера вновь обратимся к отечественной истории XX в., где вначале явно отражалась модернизационная тенденция перехода от аграрного общества к индустриальному. Противоречия этого перехода выразились в феномене революции и обусловили переход от Российской Империи к Советскому Союзу в институциональном плане, а попутно - практически полностью изменили систему идентичностей: от этнических и сословных они перешли к национальным, а конфессиональные идентичности оказались в значительной части утраченными. После разрешения противоречий модернизации к 50-м гг. СССР перешел в режим стабильного воспроизводства социокультурной системы. Однако позднее, уже в 70-е гг., обозначился новый кризис, связанный с падением отдачи инвестиций, научно-техническими инновациями и изменениями в общественном сознании уже зрелого индустриального городского типа общества. Именно в рамках этого кризиса стали одновременно развиваться и новые модернизационные установки, и установки архаизаторские [5]. Аналогично и на уровне частных факторов динамики системы можно выделить несколько обобщенных типов их влияния. Поскольку этот уровень рассмотрения касается отношений между сообществами, можно обозначить возможные общие направления этих отношений - на интеграцию, инкорпорацию или сепарацию. Интеграция в этом смысле будет оценкой установок сообществ на взаимное объединение, такое, что они выступают вариациями большой общности, соответственно, их идентичности 
также будут представлять собой вариацию общей идентичности. Инкорпорацией будет выступать склонность сообществ к включению в систему общности при сохранении собственной самостоятельности. Сепарация - установка на исключение сообщества из системы. Очевидно, что эти установки, как и в случае с факторами фундаментальной динамики, могут по-разному проявляться в разных сообществах: одни будут склонны к интеграции, другие - к инкорпорации, третьи - к сепарации. В стабильном состоянии эти ориентации скомпенсированы и, как правило, доминируют интеграция и инкорпорация, в нестабильном - активно начинает развиваться установка на сепарацию и инкорпорацию, поскольку элементы системы, сообщества начинают не только рефлексировать само нестабильное состояние, но и в любом случае негативно его оценивать. Более того, элементы, ориентированные на разные виды нестабильной динамики, будут сепаратно настроены относительно друг друга. Аналогично для того, чтобы оценить общую ситуацию в общности, следует ориентироваться на некоторую равнодействующую, складывающуюся из установок частных сообществ системы.

Опять же обращаясь к советскому периоду отечественной истории, можно отметить в этом плане, что в целом модель национальных отношений имела характер инкорпорационный, что выражалось в форме национальнотерриториальных республик и автономий СССР. Однако, как уже отмечалось выше, к 60-м гг. стала складываться интеграционная модель, на которую опиралось союзное руководство и которая выразилась в тезисе о «новой исторической общности, советском народе». Эта общность мыслилась многонациональной, однако упор делался на общих ценностях, образе жизни, политико-идеологических установках и т.п., которые полагались общими для всех советских людей. Следует отметить, что в данном случае нет смысла разделять и противопоставлять идеологическую установку советского руководства и общественные настроения, поскольку данная установка вполне была воспринята советским народом и обнаруживается даже сейчас. Параллельно этой модели стала складываться и обратная ей сепарационная установка, постулировавшая отделение национальных и республиканских сообществ от союзного центра и советского общества в целом, которой в последующем воспользовалось руководство союзных республик при распаде СССР и которая породила череду национальных конфликтов в конце перестройки и на постсоветском пространстве.

Следует оговориться, что это именно отношения общностей, а не только их представителей или каких-то организаций, установки группового и коллективного сознания членов этих сообществ. Именно поэтому и следует выделять следующий уровень динамики системы идентичностей - актуальный, на котором в качестве факторов выступают организованные группы и структуры, обеспечивающие функционирование идентичностей. Разделяя сообщества и организации, необходимо отметить, что, с одной стороны, организации всегда существуют в рамках сообществ и выступают их некоторой рефлексивной частью, а с другой - сообщества ориентируются на различные организации в их составе в зависимости от того, насколько транслируемые ими установки находят отклик и поддержку у соответствующих сообществ. На этом уровне можно относительно наглядно показать не только внешние отношения между сообществами - хотя по большей части именно на этом со- 
средоточиваются оргструктуры. Здесь анализу могут быть подвергнуты обеспеченность данной идентичности институционально-дискурсивными структурами, внутренние субидентичности, уровень их рефлексивности и адекватности как собственному положению, так и состоянию системы, внешнему окружению и т.п.

Сообщества и их подгруппы могут проявлять три варианта ориентаций, касающихся идентичности: самовоспроизводство в наличных условиях, проблематизацию собственного положения и формирование конфликтности в отношении других сообществ или общности в целом. Аналогично ранее рассмотренным примерам это может относиться как к внутренним структурам сообществ, так и к сообществам как таковым и общности в целом. В то же время нельзя не подчеркнуть, что в сообществах всегда будут подгруппы, которые проблематизируют статус и склонны к противопоставлению остальным сообществам и все зависит от уровня их поддержки со стороны аудиторий данного сообщества: если основная масса членов сообщества удовлетворена наличным положением, она не будет поддерживать проблематизирующие и конфликтные группы, и наоборот.

В этом смысле можно рассмотреть кризис национальных отношений позднего СССР как наглядную иллюстрацию взаимодействия частного и актуального уровней динамики на примере того, как под действием активности организаций с «национальной (украинской, грузинской, даже русской) антисоветской» идеологией менялся настрой советских наций с инкорпоративного на сепарационный. В условиях современного Башкортостана схожие отношения можно проследить, например, в конфессиональных сообществах активное противостояние суфийских и салафитских групп [6] уже в определенной мере влияет на ориентацию идентичностей мусульманской уммы республики [7], хотя еще рано говорить, что образовался некоторый общий вектор.

Понятно, что при любом состоянии общности, образуемом фундаментальной динамикой, все три варианта актуальной динамики будут реализовываться, но будут делать это по-разному. В общем виде установка на самовоспроизводство сообществ в стабильной системе будет основной, а остальные так или иначе будут подавляться структурами обеспечения упорядоченности. Но в нестабильной системе эта же установка в случае модернизационной динамики будет выступать инерционным, а позднее - архаическим фактором; в случае архаизирующей динамики, наоборот, фактором стабилизации отношений. Проблематизация статуса, по сути, выступает исходной посылкой для любых изменений отношений между сообществами, поскольку ставит под сомнение эти отношения и предполагает некоторую их трансформацию. В этом смысле проблематизация неизбежна в условиях нестабильной системы и можно сказать, что сама ведет к дестабилизации. В свою очередь, установка на конфликт также свойственна нестабильной системе и выражается в условиях, когда проблематизированные статусы не удалось согласовать на новом, приемлемом для сторон уровне.

В целом социокультурная динамика представляет собой равнодействующую, образуемую всей совокупностью отмеченных факторов, и будет выражаться как во внутреннем перераспределении установок сообществ, так и в смене соотношений типов их идентичностей, что, в свою очередь, будет ска- 
зываться на изменении отношений между элементами системы идентичностей общности.

Так, дестабилизация социокультурной системы будет означать, что аудитории во все возрастающем объеме оказываются неудовлетворенными наличным положением - и либо стремятся восстановить прежние отношения, либо создать некоторые новые. Это неизбежно сдвигает режимы функционирования идентичностей от инерционного к стабильному, а от него - к акцентированному, поскольку проблематизация статуса вызывает рефлексию собственного положения и самосознания. В свою очередь, это усиливает установки на инкорпорацию, по мере развития конфликтности - на сепарацию сообщества, что сказывается на статусном конфликте между фокусной и вторичными идентичностями. Последние пересматривают свое отношение к первой в пользу большего дистанцирования, а первая, стремясь сохранить свое положение, усиливает давление на вторые, подчиняя, а далее - подавляя их.

В целом в этих условиях комплементарные отношения между идентичностями системы сдвигаются в сторону напряженных, а связи между ними ослабляются, несмотря на высокую степень взаимной диффузии их содержания (образов, маркеров, стереотипов поведения и т.п.). Можно даже утверждать, что в таких условиях чем больше будет диффузия, тем больше будут акцентироваться отличия и с тем большей настойчивостью они будут отстаиваться, что в значительно меньшей степени будет способствовать конструктивному разрешению противоречий и нормализации отношений.

\section{Лumepamypa}

1. Костина А.B. Кризис современной идентичности и доминирующие стратегии идентификации в границах этноса, нации, массы // Религия, культура, образование. 2009. №4. С. 167175.

2. Федотова Н.Н. Глобализация и изучение идентичности // Знание, понимание, умение. 2011. № 1. C. $72-80$.

3. Нации и национализм / Б. Андерсон, О. Бауэр, М. Хрохидр; пер с англ. Л.Е. Переяславцевой, М.С. Панина, М.Б. Гнедовского. М.: Праксис, 2002. 416 с.

4. Буранчин А.М., Вахитов Р.Р., Демичев И.В. Социокультурные аспекты модернизационных процессов в Республике Башкортостан. Уфа: Дизайн-пресс, 2014.

5. Абдрахманов Д.М., Буранчин А.М., Демичев И.В. Архаизация российских регионов как социальная проблема. Уфа: Мир печати, 2016. 404 с.

6. Бердин A.T. Тенденции и проблемы развития современной уммы Башкортостана: фактор салафизма // Социум и власть. 2016. № 3 (59). URL: http://cyberleninka.ru/article/n/tendentsii-iproblemy-razvitiya-sovremennoy-ummy-bashkortostana-faktor-salafizma (дата обращения: 23.06.2017).

7. Юсупов Ю.М., Бердин А.Т. К вопросу о структуре мусульманской уммы: религиозные группы в Республике Башкортостан // Проблемы востоковедения. Уфа. 2017. № 1. С. 30-35. tion)

Demichev Ilya $\boldsymbol{V}$. Institute of strategic research of Republic Bashkortostan (Ufa, Russian Federa-

E-mail: senmerv@mail.ru

DOI: $10.17223 / 1998863 \mathrm{X} / 41 / 12$

DYNAMICS OF THE COMPLICATED SYSTEM OF IDENTITIES

Key words: identity, dynamics of identity, constructivism.

The article is devoted to the analysis of factors of the dynamics of group identity. Identities are considered in the frame of theory of constructivism and structural-functional approach according which they presented as complicated system of identities divided into types, forms and modes of functioning. Three groups of factors affect on the dynamics of this system. First group of factors are identi- 
fied as fundamental and they refer to the general conditions of functioning of identities. Second group of factors (particular) applies to the existence, functioning and transformation of communities which are the part of the general one and result from their interrelations. Third group of factors - topical - are the most inconstant and formed peculiarly from institutional-discursive structures of communities and society as a whole as well as from reaction of to this phenomenon of carriers of identity. It is necessary to analyze these three groups of factors altogether because each of them is identified through other and has an impact on each other. These three groups of factors can form several trends of dynamics of the system of identities. On a fundamental level stable and unstable state of the system can be distinguished. Unstable state of the system is divided into two types - "modernizational" and "archaizational". The differences between them is based on the directions of modification where first is widening of potential of the system and the second is its reduction. Similarly on the level of particular factors of the dynamics of the system some generalized types of them can be distinguished. Since on this level interrelations of communities are analyzed, the trends of this relations can be identified - integration, incorporation and separation. On topical level communities and its sub-groups can develop three options of state: self-reproduction in present conditions, dissatisfaction with own state and generating conflict towards other communities and society as a whole. As a whole sociocultural dynamics represent reluctant which made by totality of described factors and will express in both situations - in internal redistribution of attitudes of communities and in shifts of types of identities. This all in its turn will affect on changes of relations among the elements of the system of identities.

\section{References}

1. Kostina, A.V. (2009) Krizis sovremennoy identichnosti i dominiruyushchie strategii identifikatsii $\mathrm{v}$ granitsakh etnosa, natsii, massy [The crisis of modern identity and the dominant identification strategies within the borders of the ethnos, nation, masses]. Religiya, kul'tura, obrazovanie. 4. pp. $167-175$.

2. Fedotova, N.N. (2011) Globalisation and study of identity. Znanie, ponimanie, umenie Knowledge, Understanding, Skill. 1. pp. 72-80. (In Russian).

3. Anderson, B., Bauer, O. \& Hrochidr, M. (2002) Natsii i natsionalizm [Nations and Nationalism]. Translated from English by L.E. Pereyaslavtseva, M.S. Panin, M.B. Gnedovsky. Moscow: Praksis.

4. Buranchin, A.M., Vakhitov, R.R. \& Demichev, I.V. (2014) Sotsiokul'turnye aspekty modernizatsionnykh protsessov $v$ Respublike Bashkortostan [Sociocultural aspects of modernisation processes in the Republic of Bashkortostan]. Ufa: Dizayn-press.

5. Abdrakhmanov, D.M., Buranchin, A.M. \& Demichev, I.V. (2016) Arkhaizatsiya rossiyskikh regionov kak sotsial'naya problema [Archaization of Russian regions as a social problem]. Ufa: Mir pechati.

6. Berdin, A.T. (2016) Tendencies and problems of the development of present-day Bashkortostan Ummah: A factor of Salafism. Sotsium i vlast' - Society and Power. 3(59). [Online] Available from: http://cyberleninka.ru/article/n/tendentsii-i-problemy-razvitiya-sovremennoy-ummy-bashkortostanafaktor-salafizma. (Accessed: 23rd June 2017). (In Russian).

7. Yusupov, Yu.M. \& Berdin, A.T. (2017) K voprosu o strukture musul'manskoy ummy: religioznye gruppy v Respublike Bashkortostan [On the structure of the Muslim Ummah: Religious groups in the Republic of Bashkortostan]. Problemy Vostokovedeniya - The Problems of Oriental Studies. 1. pp. $30-35$. 